\title{
Antibiotic Susceptibility Pattern of Bacterial Isolates Causing Wound Infection Among the Patients Visiting B \& B Hospital
}

\author{
Jaya Krishna Yakha ${ }^{1}$, Amit Raj Sharma ${ }^{2}$ Nawaraj Dahal ${ }^{4}$, Binod Lekhak ${ }^{3}$ and Megha \\ Raj Banjara ${ }^{3}$ \\ ${ }^{I}$ GoldenGate International College, Kathmandu, Nepal \\ ${ }^{2}$ Nepal Academy of Science and Technology, Lalitpur, Nepal \\ ${ }^{3}$ Central Department of Microbiology, Tribhuvan University, Kathmandu,Nepal \\ ${ }^{4} B$ \& B Hospital, Lalitpur, Nepal \\ e-mail jkyakha2012@gmail.com
}

\begin{abstract}
A wound is any physical injury involving a break in the skin, and exposed subcutaneous tissues provide a favorable substratum for a wide variety of microorganisms to contaminate and colonize. In this study a total of 870 plus samples were collected from patients visiting B \& B hospital suspecting wound infection and then analyzed. The causative agents were isolated, identified by culture and biochemical tests and their susceptibility pattern to antibiotics were determined by using CLSI guidelines. Out of total samples, 476(44.8\%) showed bacterial growth. Among growth cases $22.9 \%$ were mixed growths. Among all bacterial isolates, 12 species were identified of them, $70.6 \%$ were Gram-negative and 29.4\% were Gram-positive. Among Gram-positive isolates, Staphylococcus. aureus (74.2\%) was most common followed by CoNS (8.6\%), Non haemolytic Streptococci (8.6\%), Enterococcus spp. (4.3\%) and $\beta$-haemolytic Streptococci (4.3\%). Among Gram-negative bacteria, the most common isolate was Pseudomonas aeruginosa (31.5\%) followed by E.coli (24.8\%), Acinetobacter spp. (14.6\%), Enterobacter spp. (14.0\%) and Klebsiella spp. (13.4\%). Proteus spp. and Citrobacter spp. were less common.With regard to AST pattern, $S$. aureus was most susceptible to chloramphenicol and highly resistance to penicillin. Among them, 7.7\% were MRSA. For other Gram-positive isolates as well, effective drug was found to be chloramphenicol. For P. aeruginosa, the most effective drug was imipenem $(94.3 \%)$ followed by amikacin (63.2\%). Likewise, for other Gram-negative bacteria most effective drug was imipenem followedby amikacin. Among the total P. aeruginosa isolates, 58.5\% were found to be MDR. For all these $P$. aeruginosa isolates MIC on gentamycin and ciprofloxacin illustrate the simultaneous presence of ciprofloxacin resistance and reduced gentamycin susceptibility. These results indicated that antibiotic resistance in Gram-positive and Gram-negative bacteria were increasing in alarming trend that leads to the failure of treatment.
\end{abstract}

Key words: wound infection, multidrug resistannce (MDR), minimum inhibitory concentration (MIC)

\section{Introduction}

A wound is a breach in the skin and the exposure of subcutaneous tissue following loss of skin integrity providing moist, warm and nutritive environment that is conducive to microbial colonization and proliferation (Bowler et al. 2001). Wound can be infected by a variety of microorganisms ranging from bacteria to fungi and parasites as well as virus (Church et al. 2006). The most common organisms are Staphylococcus aureus,, Pseudomonas aeruginosa, Escherichia coli, Klebsiella species and Acinetobacter species. (Gupta et al. 2002, Eselbelahie et al. 2013). The control of wound infections has become more 
challenging due to widespread bacterial resistance to antibiotics, and to a greater incidence of infections caused by methicillin-resistance $S$. aureus, polymicrobic flora (Shittu et al. 2002).

The present study was conducted to determine the prevalence of MDR $P$. aeruginosa, antibiotic susceptibility pattern of isolates and MIC for $P$. aeruginosa to provide guidelines on suitable treatment and ensure infection-control policies leading to appropriate treatment of wound infection and prevention of emergence of antimicrobial resistance.

\section{Methodology}

A exploratory study was carried out at the Microbiology Laboratory of B \& B Hospital, Lalitpur. Required samples (wound Pus/swab) obtained from the patient visiting $\mathrm{B}$ and $\mathrm{B}$ hospital were received in the laboratory within 30 minutes of collection. The samples were collected following aseptic technique which then were macroscopically analysed for their color and odor and microscopically analysed for type and morphology of bacteria. The samples were inoculated on blood agar (BA), Mac-conkey agar (MA), chocolate agar (CA) and mannitol salt agar (MSA) using standard protocol (Isenberg 2004). Macconkey agar plates were incubated aerobically, while BA and CA plates were incubated in microaerophilic condition at $37^{\circ} \mathrm{C}$ for 24 to 48 hours in incubator. All the isolates were identified by colony morphology, staining reaction and the biochemical properties (Cheesbrough 2006). The antimicrobial susceptibility test of isolates was performed by Modified Kirby Bauer disc diffusion method using the standard guidelines and interpretive criteria of the CLSI (2013). After this isolated $S$. aureus was screened for methicillin resistance using cefoxitin disc $(30 \mu \mathrm{g})$ as per standard guidelines provided by CLSI, (2013). And MDR $P$. aeruginosa was identified as resistant to $\geq 3$ antimicrobial classes (Magiorakos et al. 2011). MICs of ciprofloxacin and gentamycin were determined by agar dilution method (EUCAST 2000, Andrews 2001) and the guidelines of the Clinical and Laboratory Standards Institute (CLSI 2013). The tests were performed by making a series of antibiotic concentrations on Mueller-Hinton agar plates. A reference strains, E.coli ATCC 25922 and S. aureus ATCC 25923 were used as control. All the data were entered in SPSS version 20 and WHONET 5.6. Statistical analyses were done using the same software.

\section{Results and Discussion}

Of 870 sample processed, 390 (44.1\%) showed their growth. Among growth positive cases, 304(77.9\%) were monoisolates while $86(22.1 \%)$ had polymicrobial growth. $(56 \%)$. High rate of growth positive rate in infection was found in case of male gender than female gender, i.e. $46.9 \%$ in male and $37.4 \%$ in female, which was found to be statistically significant (p-value < $0.05)$. Among total growth, the highest growth rate was found in age group 21-30 (25.4\%), followed by $31-40(16.2 \%)$ and $11-20(16.2 \%)$. Least growth was found in an age group of $0-10$ and above 60 years. A regard to the location of patients, high growth was found in indoor patients (54.9\%) than outdoor (OPD) patients $(38.8 \%)$ and emergency patients $(20.3 \%)$.

Out of 476 growth positive wound swab samples, Gram negative bacteria were found $70.6 \%$, while Gram positive bacteria in $29.4 \%$. Among the gram negative isolates, $P$. aeruginosa $106(31.5 \%)$ was the most predominant followed by E.coli $82(24.4 \%)$, Acinetobacter spp. 49(14.6\%). Enterobacter spp. $47(14.0 \%)$ and Klebsiella Spp 45(13.4\%). Other bacteria like Proteus spp. 6(1.8\%) and Citrobacter Spp $1(0.3 \%)$ were lower in a number. Similarly, among Gram positive bacteria, S. aureus 104 (74.2\%) was the most common isolates followed by CoNS 12 (8.6\%), NHS $12(8.6 \%)$ Enterococcus spp. and $\beta$ Haemolytic Streptococci.

Among 104 S. aureus, the resistance pattern showed that the most effective antibiotics were chloramphenicol $(4.8 \%)$ followed by clindamycin $(7.7 \%)$ and cefoxitin(7.7\%) while highly resistant to Penicillin(99.1\%) and amoxicillin(86.5\%), (Table 1).

Table 1. Antimicrobial susceptibility pattern of the Staphylococcus aureus

\begin{tabular}{l|l|l}
\hline Antibiotics & Resistance & Total \\
\hline Ciprofloxacin & $42(40.4 \%)$ & 104 \\
Ofloxacin & $41(39.4 \%)$ & 104 \\
Gentamycin & $42(40.4 \%)$ & 104 \\
Ceftriaxone & $13(22.1 \%)$ & 104 \\
Chloramphenicol & $5(4.8 \%)$ & 104 \\
Cefoperazone/Sul & $10(9.6 \%)$ & 104 \\
Penicillin & $103(99.1 \%)$ & 104 \\
Amoxicillin & $90(86.5 \%)$ & 104 \\
Erythromycin & $35(33.6 \%)$ & 104 \\
Cefoxitin & $8(7.7 \%)$ & 104 \\
Clindamycin & $8(7.7 \%)$ & 104 \\
\hline
\end{tabular}




\section{P. aeruginosa}

Among 106 P. aeruginosa isolates, imipenem (5.7\%) was found to be most effective followed by amikacin $(36.8 \%)$ and highly resistant against the ceftazidime (73.6\%), (Table 2).

Table 2. Antimicrobial susceptibility pattern of $\boldsymbol{P}$. aeruginosa

\begin{tabular}{l|l|l}
\hline Antibiotics & Resistance & Total \\
\hline Ciproloxacin & $55(51.9 \%)$ & 106 \\
Ofloxacin & $55(51.9 \%)$ & 106 \\
Gentamycin & $57(53.8 \%)$ & 106 \\
Amikacin & $39(36.8 \%)$ & 106 \\
Ceftazidime & $78(73.6 \%)$ & 106 \\
Chloramphenicol & $63(59.4 \%)$ & 106 \\
Cefoperazone/Sul & $48(45.3 \%)$ & 106 \\
Meropenem & $55(51.9 \%)$ & 106 \\
Imipenem & $6(5.7 \%)$ & 106 \\
Piperacillin/Taz & $56(52.8 \%)$ & 106 \\
\hline
\end{tabular}

E. coli

Among 82 E.coli isolates, imipenem (3.6\%) was found the most effective drug followed by amikacin $(13.4 \%)$ and highly resistant against ciprofloxacin $(54.9 \%)$ and ofloxacin $(54.9 \%)$, (Table 3).

Table 3. Antimicrobial susceptibility pattern of the E. coli.

\begin{tabular}{l|l|l}
\hline Antibiotics & Resistance & Total \\
\hline Ciproloxacin & $45(54.9 \%)$ & 82 \\
Ofloxacin & $45(54.9 \%)$ & 82 \\
Gentamycin & $23(28.1 \%)$ & 82 \\
Amikacin & $11(13.4 \%)$ & 82 \\
Ceftriaxone & $56(68.3 \%)$ & 82 \\
Chloramphenicol & $17(20.7 \%)$ & 82 \\
Cefoperazone/Sul & $14(17.1 \%)$ & 82 \\
Meropenem & $11(13.4 \%)$ & 82 \\
Imipenem & $3(3.6 \%)$ & 82 \\
Piperacillin/Taz & $24(29.3 \%)$ & 82 \\
\hline
\end{tabular}

\section{Prevalence of MDR $P$. aeruginosa}

Among the total P. aeruginosa isolates, 62 were found to multi drug resistance, of which 2 were resistant to 3 antimicrobial classes. Among MDR isolates, 7 were resistant to three antimicrobial classes; 4 were resistant to 4 classes; 12 were resistant to 5 antimicrobial classes; 6 were resistant to 6 antimicrobial classes and 33 were resistant to 7 antimicrobial classes.

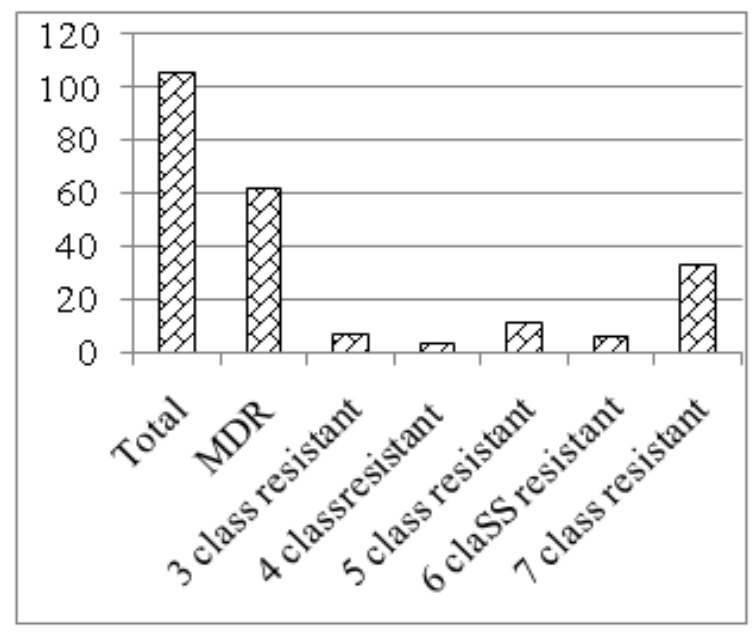

Fig.1. MDR antimicrobial class resistant of $P$. aeruginosa.

MIC on gentamycin and ciprofloxacin of P. aeruginosa

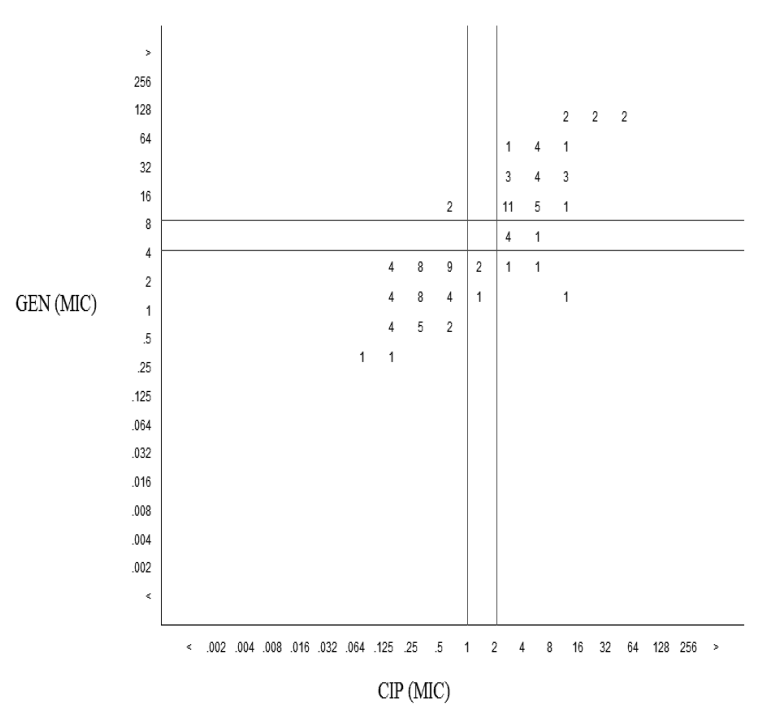

Fig. 2. MIC of P. aeruginosa (gentamycin vs ciprfloxacin)

In our study, out of 870 samples from patients with wound infection, $44.9 \%$ samples showed positive growth. In similar studies conducted by Karki (2011) in pediatric cases, Shrestha and Basnet (2009) showed growth positive were $47 \%$ \& $50 \%$ respectively. A study conducted by Giacometti et al. (2000), Manyahi (2012) in the tertiary hospital, more than $90 \%$ show growth. Culture negative results could be due to non infective bacteria, manual error in collection, transportation, culture media and collection of sample from patient taking antibiotic. 
Among growth cases, $77.9 \%$ cases showed single isolates and $22.1 \%$ cases showed multiple isolates. The result agreed with the study carried out by Fadeyibi $e t$ al., (2012) and Manyahi (2012). The growth was found to be higher in male patients $46.9 \%$ than in female patients $(37.4 \%)$. Similar study carried out by Parajuli (1997) agree with our result. The relatively higher percentage of male patients might be due to active involvement of males in outdoor activities, including agricultural work resulting into high infection and prevalence of high rate of accidental cases among them (Naik \& Deshpande 2011).

Among total growth of $44.9 \%$, the highest growth rate was found in age group 21-30 (25.4\%), followed by $31-40(16.2 \%)$ and $11-20(16.2 \%)$. Least growth was found in an age group of $0-10$ and above 60 years. Our findings agree with the studies carried out by Kumari (2008) and Akinjogunla et al. (2009). The reason behind this age group (21-30) showed high growth among all age categories may be due to high prevalence of this age group in our study as well as the group being active age group or working age group hence the chances of occurring accidents leading to hospital admission.

The high rate of growth was found to be in indoor patients $(54.9 \%)$ than OPD patients $(38.8 \%)$ and emergency patients $(20.3 \%)$, which was similar to the study of Iregbu et al. (2013). The reason for the high prevalence in ward may be due to factor associated with acquisition of nosocomial pathogens in patients with recurrent long term hospitalization, complicating illness, prior administration of antimicrobial agents.

In our present study, out of total bacterial isolates, $70.6 \%$ were Gram negative and $29.4 \%$ were Grampositive bacteria. In a similar study conducted by Giri et al. (2008), Iregbu et al. (2013), Eselbelhie et al. (2013) Gram negative bacteria were found predominant. Most of the hospital-based studies nowadays show that Gram-negative bacteria was more prevalent than Gram-positive bacteria. Banjara et al.(2002) at TUTH showed that high rate of Gramnegative bacteria are found in HAI.

Among these isolated pathogens $P$. aeruginosa being the most predominant $(22.3 \%)$ occurring in total isolates and $31.5 \%$ of Gram-negative bacteria while S. aureus was the second predominant isolate among the total isolates. In a similar study conducted by Thanni et al. (2003), P. aeruginosa was most predominant one $(29.9 \%)$ among the total isolates while $S$. aureus was predominant $(27.5 \%)$ among Gram positive isolates. The study conducted by Iregbu et al. (2013) gram-negative bacilli constituted $66 \%$ of all the pathogens with $P$. aeruginosa as the most frequent (19\%). Higher prevalence of $S$. aureus among Gram-positive bacteria and $2^{\text {nd }}$ predominant isolates among the total, seen in this study was also reported by other researchers, Mehta et al. 2000 and Mohammad et al. 2013. Many researchers said that most commonly isolated. S. aureus was ubiquitous and the most common cause of localized suppurative lesions in human beings.

With regard to antibiotic susceptibility pattern, the most effective antibiotic against $S$. aureus was chloramphenicol $(4.8 \%)$ followed by clindamycin $(7.7 \%)$, penicillin $(99.1 \%)$ and amoxicillin $(86.5 \%)$ was least effective. These results agree with Shrestha, (2009). Other developing countries, also reported high resistance rates of $S$. aureus to amoxicillin and penicillin (Anguju et al. 2007).

In a present study, $7.7 \%$ of $S$. aureus isolates were MRSA, and emerged as a major pathogens worldwide. Studies on MRSA have shown their wide variation. Naik and Deshpande (2011) showed 8.0\% of MRSA which agree to our study. Chen and Zhang 2007, at China showed $(82.5 \%)$. P. aeruginosa was most effective against imipenem $(5.7 \%)$ followed by amikacin $(36.8 \%)$. The least effective antibiotic was ceftazidime $(73.6 \%)$. This was agreed by Mahmood, 2000 , towards amikacin $(92 \%)$ followed by meropenem and imipenem (88\%) sensitive. Rajput et al (2008), in which Pseudomonas isolated from burn wound was less resistant to imipinem (26\%) and highly resistant to ceftazidime (70\%). For E.coli, imipenem $(3.6 \%)$, amikacin $(13.4 \%)$ and meropenem $(13.4 \%)$ were considerably effective while ceftriaxone $(68.3 \%)$ and ciprofloxacin $(54.9 \%)$ and ofloxacin $(54.9 \%)$ were least effective. This was supported by Mahmood (2000) and where E. coli was found to be $100 \%$ sensitive to imipenem.

Resistance to antibiotics antimicrobial agents is a major concern worldwide and is exemplified by the global spread of MRSA, MDR $P$. aeruginosa, Acinetobacter spp. In our study out of $106 P$. 
Jaya Krishna Yakha et al/Antibiotic Susceptibility Pattern

aeruginosa isolates $58.5 \%$ were MDR. This result was supported by the studies conducted by Upadhya et al. 2012, Gupta et al. 2002, where MDR P. aeruginosa were $58.3 \%$ \& $54.8 \%$ respectively. The increase in a number of immune compromised hosts, debilitating effects of prolonged hospitalization, application of medical equipments (airways, catheters, etc.) and improper infection-control measures have contributed to the dissemination of MDR-bacteriain hospital settings (Esen \& Leblebicioglu, 2008, Furtado et al. 2009). Resistance to antimicrobial agents is a major concern worldwide and High resistance pattern of $P$. aeruginosa is major problem. All the isolates subjected for determination of MIC values of commonly used drug i. e. fluoroquinolone (Ciprofloxacin) and aminoglycoside (Gentamycin). Scattergram correlating the MICs of gentamycin and ciprofloxacin illustrated the simultaneous presence of ciprofloxacin resistance and reduced gentamycin susceptibility.

In our study Gram-negative bacteria predominated over Gram-positive bacteria. The most common isolates were P. aeruginosa S. aureus, E coli Klebsiella spp. and Acinetobacter spp. Most of the organisms, particularly P. aeruginosa, S. aureus and E.coli spp. were isolated from inpatients location were resistant to most of the antibiotic tested. S.aureus isolates were resistant to penicillin and amoxicillin, whereas $P$. aeruginosa, Acinetobacter spp., E. coli and Klebsiella spp. were resistant to ciprofloxacin, ofloxacin and resistance to third generation cephalosporin. These results indicate that antibiotic resistance pattern in Gram-positive and Gram-negative bacteria are increasing in alarming trend that leads to the failure of treatment.

\section{Acknowledgments}

We greatly acknowledge the support provided by B \& B Hospital and the Golden Gate International College, Kathmandu Nepal.

\section{References}

Akinjogunla, O. J., Adeko, A.A., Mboto, C.I., Chukwudebelu I.C. and Udokang I.p. 2009. Bacteriology of framycetin and silver sulphadiazine as topical agents for burn. A pilot study. Burns.35: 672-676.

Andrews, J.M 2001. Determination of minimum inhibitory concentrations. Journal of Antimicrobial Chemotherapy Suppl. 48, SL, 5-16.
Anguzu, J.R. and D. Olila.2007. Drug sensitivity patterns of bacterial isolates from septic post-operative wounds in a regional referral hospital in Uganda. Afr. Health Sci. 7:148-154.

Banjara, M.R., A.P. Sharma, A.B. Joshi, N.R. Tuladhar, P. Ghimire and D.R. Bhatta. 2003. Surgical wound infection in Tribhuvan University Teaching Hospital. Journal of Nepal Health Research Council 1: 41-45.

Boweler, P.G., B.I. Duerden and D.G. Armstrong. 2001. Wound microbiology and associated approaches to wound management. Clin.Microbiol.Rev. 14: 244-26.

Cheesbrough M (2006). District Laboratory Practice in Tropical Countries. $2^{\text {nd }}$ edn. Cambridge. Newyork, Melbourne, Madrid, Cape Town, Singapore, Sao Poulo: Cambridge University Press.

Church, D., S. Elsayed, O. Reid, B. Winston and R. Lindsay. 2006. Burn wound infection. Clin. Microbiol. Rev. 19: 403-434.

CLSI. 2013. Performance standards for antimicrobial susceptibility testing. Twenty-Third Informational Supplement.33:1-199.

Esebelahie, N.O., F.O. Esebelahie and R. Omoregie. 2013. Aerobic bacterial isolates from wound infection. Afr. J. Cln. Exper. Microbiol. 14: 155-159.

Esen,S. and H. Leblebicioglu. 2004. Prevalence of nosocomial infections at intensive care units in Turkey: a multicentre 1-day point prevalence study.Scand $J$. Infect. Dis. 36: 144-148.

European Committee for Antimicrobial Susceptibility Testing: 2000. Determination of minimum inhibitory concentrations (MICs) of antibacterial agents by agar dilution. Eucast Definitive Document. E. Def 3.1.

Fadeyibi, I.O., Raji, M.A., Ibrahim, N.A., Ugburo, A.O. and Ademilu, S. 2012. Bacteriological profile of infected burn wounds in burn wards of teaching hospital in Southwest Nigeria.

Furtado, G.H., M.D. Bergamasco, F.G. Menezes, D. Marques, A. Silva, L.B. Perdiz, and E.A. 2009. Imipenem-resistant $P$. aeruginosa infection at a medical-surgical intensive care unit: Risk factors and mortality.J. Crit. Care. 24: 625.

Giocometti, A., O. Cirioni, A.M. Schimizzi, M.S. Del, F. Prete, M.M.D Barchiesi, E. Errico, G. Petrelli and J. ScaliSe. 2000. Epidemiology and microbiology for surgical wound infection. J. Clin. Microbiol. 38: 918922.

Gupta, N., V. Gautam, S. Saini, L. Singh, and D.R. Arora.2002. Prevalence of multi drug organism in wound infection. J. infect. Dis. Antimicrobial. Agent. 19: 111-117.

Iregbu, K.C., N.S. Uwaezuoke, I.P. Nwajiobi-Princewill, S.O, Eze, N. Medugu, S. Shettima and Z, Modibbo. 2013. A profile of wound infecgtion in national hospital Abuja. Afr. J. Cln. Exper. Microbio. 14: 160-163. 
Nepal Journal of Science and Technology Vol. 15, No.2 (2014) 91-96

Kumari, K. 2008. Pattern of bacterial isolates and antibiogarm from open wound infection among the indoor patients of Bir Hospital. M. Sc. dissertation presented to the Central Department of Microbiology, Tribhuvan University, Kathmandu, Nepal.

Magiorakos, A.P., Srinivasan A., Carey, R.B., Carmeli Y., Falagas, M.E., Giske C.G., Harbarth S., Hindler, J.F., G. Kahlmeter, Olsson-Liljequist B., Paterson D.L., Rice L.B., Steling J., Struelens, M.J., Vatopoulos, A., Weber, J.T. and Monner, D.L. 2012. Multidrugresistant, extensively drug resistant and pandrugresistant bacteria: an international expert proposal for interim standard defination for acquired resistance. Clin. Microbiol. Infect. 18: 268-281.

Mahmood, A. 2000. Bacteriology of surgical site infection and antibiotic susceptibility pattern of isolates in a tertiary care hospital at Karachi. JPMA. 50:256-263.

Manyahi, J. 2012. Bacteriological spectrum of post operative wound infections and their antibiogram in a tertiary hospital, Dares Salaam, Tanzania. Master thesis in Medicine (Microbiology and Immunology), Muhimbili University of Health and Allied Sciences.

Mehta, P.A., C.K. Cunningham, L.B. Colella, G. Alferis and L.B. Weiner. 2000. Risk factors for sternal wound infection and other infection in pediatric cardiac surgery patients. J. Pediatr. Infect. Dis. 19: 1000-1004.

Mohammed, A., G.O. Adeshina, and Y.K.E. Ibrahim. 2013. Retrospective incidence of wound infections .and antibiotic sensitivity pattern: A study conducted at the Aminu Kano Teaching Hospital, Kano, Nigeria. International Journal of Medicine and Medical Sciences 5: 60-66.
Naik, G. and S.R. Deshpande. 2011. A study on surgical site infections caused by Staphylococcus aureus with a special search foe methicillin-resistant isolates. J. Clin. and Diag. Research. 5:502-508.

Parajuli, K. 1997. Study of causative organisms from pus samples and its antibiotic susceptibility pattern. M. Sc. dissertation presented to the Central Department of Microbiology, Tribhuvan University, Kathmandu, Nepal.

P.R. Giri, H.P. Pant, P.R. Shankar, C.T. Sreeramareddy and P.K. Sen. 2008. Surgical site infection and antibiotics use pattern in a tertiary care hospital in Nepal. JPMA 58:148-151.

Rajput, A., Singh, K.P., Kumar, V., Saxena, R and Singh R.K.. 2008. Antimicrbial resistance pattern of aerobic and anaerobic bacteria in post operative wound sepsis. Arch.surg. 121: 924-929.

Shrestha, B. and R.B. Basnet. 2009. Wound infection and antibiotic sensitivity pattern of bacterial isolates. PMJN 9: 1-6.

Shittu, A.O., D.O. Kolawole, and E.A.R. Oyedepo. 2002. Wonud infections in two health institutions in Ile Ife, Nigeria: A cohort Study. Afr. J. Biomed. Res 5:97-102.

Thanni, L.O.A., O.A. Olubunmi, and M.D. Agboola. 2003. Prevalence of bacterial pathogens in infected wound in a tertiary hospital, (1995-2001): J. Natl. Med. Assoc. 95:1189-1195.

Upadhyay, A.K., R. Maharjan and B. Shakya. 2012.Multidrug resistance bacteria in different clinical samples in National Medical College and Teaching Hospital, Birgunj, Nepal. RJPBC S3: 797-807. 